\title{
Assessment of obstruction length and optimal viewing angle from biplane $X$-ray angiograms
}

\author{
Shengxian Tu • Gerhard Koning • \\ Wouter Jukema $\cdot$ Johan H. C. Reiber
}

Received: 20 June 2009/Accepted: 1 September 2009/Published online: 18 September 2009

(C) The Author(s) 2009. This article is published with open access at Springerlink.com

\begin{abstract}
Three-dimensional quantitative coronary angiography (3D QCA) has been encouraged by the increasing need to better assess vessel dimensions and geometry for interventional purposes. A novel 3D QCA system based on biplane X-ray angiograms is presented in this paper. By correcting for the isocenter offset and by improving the epipolar constraint for corresponding two angiographic projections, accurate and robust reconstruction of the vessel centerline is achieved and the reproducibility of its applications, e.g., the assessments of obstruction length and optimal viewing angle, is guaranteed. The accuracy and variability in assessing the obstruction length and optimal bifurcation viewing angle were investigated by using phantom experiments. The segment length assessed by 3D QCA correlated well with the true wire segment length $\left(r^{2}=0.999\right)$ and the accuracy and precision were $0.04 \pm 0.25 \mathrm{~mm}$ $(P<0.01)$. 3D QCA slightly underestimated the rotation angle (difference: $-1.5^{\circ} \pm 3.6^{\circ}, P<0.01$ ),
\end{abstract}

S. Tu · G. Koning $(\bowtie) \cdot$ J. H. C. Reiber

Department of Radiology, Leiden University Medical Center, Leiden, The Netherlands

e-mail: gkoning@medis.nl

W. Jukema

Department of Cardiology, Leiden University Medical

Center, Leiden, The Netherlands

S. Tu · G. Koning · J. H. C. Reiber

Department of Applied Research, Medis Medical Imaging

Systems B.V., Leiden, The Netherlands while no significant difference was observed for the angulation angle (difference: $-0.2^{\circ} \pm 2.4^{\circ}, P=$ $0.54)$. In conclusion, the new $3 \mathrm{D}$ QCA approach allows highly accurate and precise assessments of obstruction length and optimal viewing angle from $\mathrm{X}$ ray angiography.

Keywords Bifurcation - Correspondence Isocenter offset . Optimal viewing angle . Three-dimensional reconstruction
Abbreviations
DICOM The digital imaging and communications in medicine
PCI Percutaneous coronary interventions
QCA Quantitative coronary angiography
RAO Right anterior oblique
LAO Left anterior oblique

\section{Introduction}

Accurate interpretation of vessel dimensions from $\mathrm{X}$-ray angiography is of great importance to the diagnosis of cardiovascular diseases and to support coronary interventions. Two-dimensional quantitative coronary angiography (2D QCA) has been widely used to obtain clinically relevant parameters, e.g., obstruction length and percent stenosis, and to assess the results of PCI-trials [1]. However, due to the 
perspective deformation of vessels on the projection images, 2D QCA has inherent limitations in interpreting the true dimensions of the vascular structures, resulting in an increasing interest in the research and development of three-dimensional quantitative coronary angiography (3D QCA) systems [2-6].

Restoring 3D morphology of vascular structures requires at least two projections. Biplane angiograms supply a nice solution to the reconstruction problem by allowing two acquisitions obtained at the same time. However, the reconstruction from two projections is an underdetermined problem, allowing a huge number of feasible solutions which could satisfy the projection data [5]. In addition, mechanical distortions in X-ray systems, as well as noise corruption in the projections, make the development of reliable 3D QCA systems a non-trivial task.

The accuracy of 3D QCA systems mainly depends on the reconstruction of vascular structures, of which the centerline reconstruction is the primary and yet the most important step. Once an acceptable solution for the $3 \mathrm{D}$ centerline has been obtained, the issue of the reconstruction of the cross sections becomes relevant. To determine the exact position of 3D centerline points, the correspondence between two projected centerlines should be established first, mainly by using the epipolar constraint, i.e., the constraint between a projection point and its corresponding epipolar line, being the projection of the Xray beam directed towards a particular point on one of the projection planes onto the second projection plane [5]. However, the isocenter offset, i.e., the spatial difference between the isocenters of the frontal and lateral systems, together with the small perspective projection angles for noise-corrupt centerlines, could greatly deteriorate the epipolar constraint, leading to an inaccurate correspondence.

Many efforts [2-4, 6] have been undertaken to correct for the isocenter offset, either by manually or automatically identifying several reliable features, e.g., anatomical landmarks, bifurcation points, as reference points on both projections and involving the epipolar constraint [5] to approximate the isocenter offset. At least five to eight pairs [3] or two to five pairs [7] of reference points were needed to approximate the isocenter offset. However, it may be very difficult in clinical practice to find that many reliable reference points in both projections, due to the presence of foreshortening and overlap on the coronary segments with potential features. In addition, requiring the user to indicate many reference points is not very attractive from a workflow perspective. To guarantee the accuracy and reliability in the interactive procedure has already been a difficult task.

We have been very interested in developing a fast and reliable system for vascular centerline reconstruction from X-ray angiograms. To minimize the dependency of correcting for the isocenter offset on the number of reliable reference points and yet to achieve a good correspondence in centerline reconstruction, we have found that identifying one to three pairs of reference points is sufficient to approximate the isocenter offset for the improvement of using the epipolar constraint in centerline reconstruction. In case of the presence of small perspective projection angles for noise-corrupt centerlines, the usage of epipolar constraint is further improved by building a distance transformation matrix and by searching the optimal corresponding path in the matrix. The reconstructed centerlines and cross sections can then be used to assess obstruction (stenotic lesion) length and optimal bifurcation viewing angle. In the following sections, the methodology will be presented, followed by the applications of centerline reconstruction in assessing obstruction length and optimal bifurcation viewing angle; next, the validation approach will be described, followed by the Results, the Discussions and the Conclusions.

\section{Method}

Image geometry

Conventional biplane angiographic equipment consists of a frontal X-ray system and a lateral X-ray system, with a common coordinate system. In theory, the frontal projection axis (central beam) intersects with the lateral projection axis into the so-called isocenter, and the whole X-ray system rotates around the isocenter. However, due to the system distortion caused by the gravity and mechanical influence, the isocenter could hardly be observed as a stable point [2]. Therefore, we define two isocenters, a frontal isocenter and a lateral isocenter, to explicitly model the biplane angiogram under that specific acquisition. When no system distortion is present, these two isocenters will coincide with each other. Otherwise, 
an isocenter offset is expected and this offset should be eliminated before the reconstruction of vascular centerlines.

Many sources of distortion might contribute to the isocenter offset, e.g., the gantry sag and the inaccurate reading of the acquisition angle. During many years of quality control on several X-ray systems at various hospitals, we found that gantry sag was the main reason leading to the shift of the isocenter. Due to the gravity and mechanical influence, gantry sag constantly happens during the image acquisition when the acquisition angle is adjusted. For a monoplane system, rotating the gantry to a different acquisition angle could cause a significant shift of more than $20 \mathrm{~mm}$ to its isocenter [7]. For a biplane system, either the frontal gantry or the lateral gantry could sag significantly under circumstances. The difference of gantry sags between the frontal and lateral systems could be even bigger, which would cause the frontal projection axis and the lateral projection axis not to intersect, resulting in a significant isocenter offset.

Given the aforementioned facts, we ignore insignificant sources of distortion and assume that the uneven gantry sag between the frontal X-ray system and the lateral X-ray system is the only reason accounting for the isocenter offset. By this assumption, we define the imaging geometry as one X-ray system in fixed position with a shift equal to the amount of the isocenter offset in the other X-ray system. Figure 1 shows our 3D biplane model with an isocenter offset $\mathrm{O}-\mathrm{O}^{\prime}$. The system distortion can be examined by using the epipolar constraint [5]. Due to the absence of pincushion distortion in modern X-ray image intensifiers, each projection point should intersect with its corresponding epipolar line, when no system distortion is present. However, due to the presence of the isocenter offset, the projection of reference point $A$ in frontal image intensifier, $A_{\mathrm{F}}$, does not intersect with its corresponding epipolar line. The same holds for the reference point $B$. This ill-defined epipolar constraint can significantly jeopardize its usage in corresponding the frontal and lateral projections for $3 \mathrm{D}$ centerline reconstruction.

Approximation of the isocenter offset

In order to create a good correspondence between the frontal and lateral centerlines, i.e., to enforce the centerline points to correctly intersect with their corresponding epipolar lines, the isocenter offset should be calculated and eliminated. Due to the uncertainty of gantry sag, the real amount of isocenter offset varies for different acquisitions and is not reproducible. Our solution is to use one to three pairs of reference points, chosen from some anatomical landmarks visualized on both projections, e.g., the bifurcation points, to approximate the isocenter offset.

The error of approximation is defined as the total distance from the reference points to their corresponding epipolar lines, e.g., the $A_{\mathrm{F}} M_{\mathrm{F}}$ and $B_{\mathrm{F}} N_{\mathrm{F}}$ in Fig. 1. By using the aforementioned biplane geometry, the error can be formulated as an explicit function of the isocenter offset. By minimizing the error function, the approximation of the isocenter offset is obtained. An example of correspondence before and after eliminating the isocenter offset is given by Figs. 2 and 3, respectively. Clearly, the reliability of the epipolar constraint [5] is improved and good correspondence between the two projections is established after eliminating the isocenter offset.

\section{Centerline reconstruction}

The vascular centerline is defined in this paper as the curve that passes through the center of the vessel lumen. The accuracy of the centerline reconstruction depends both on the 2D centerline extraction and on the $3 \mathrm{D}$ point reconstruction. In our approach, 2D contours are automatically detected by a validated contour detection algorithm [8] after manually specifying the start and end positions of the segment of interest on both projection images. 2D centerlines are then extracted from the contours and used to reconstruct the 3D centerline points.

The $3 \mathrm{D}$ point reconstruction algorithm requires the knowledge of correspondence between the frontal and lateral centerlines. This knowledge can be facilitated by using the epipolar constraint [5]. However, an ill-defined epipolar line due to the system distortion could cause significant error in the correspondence. Multiple intersections of the 2D centerline and the epipolar line, as well as noise corruption in the centerline, could further deteriorate the correspondence. An example of the possible difficulties in creating correspondence by using the epipolar constraint is given by Fig. 4 . 


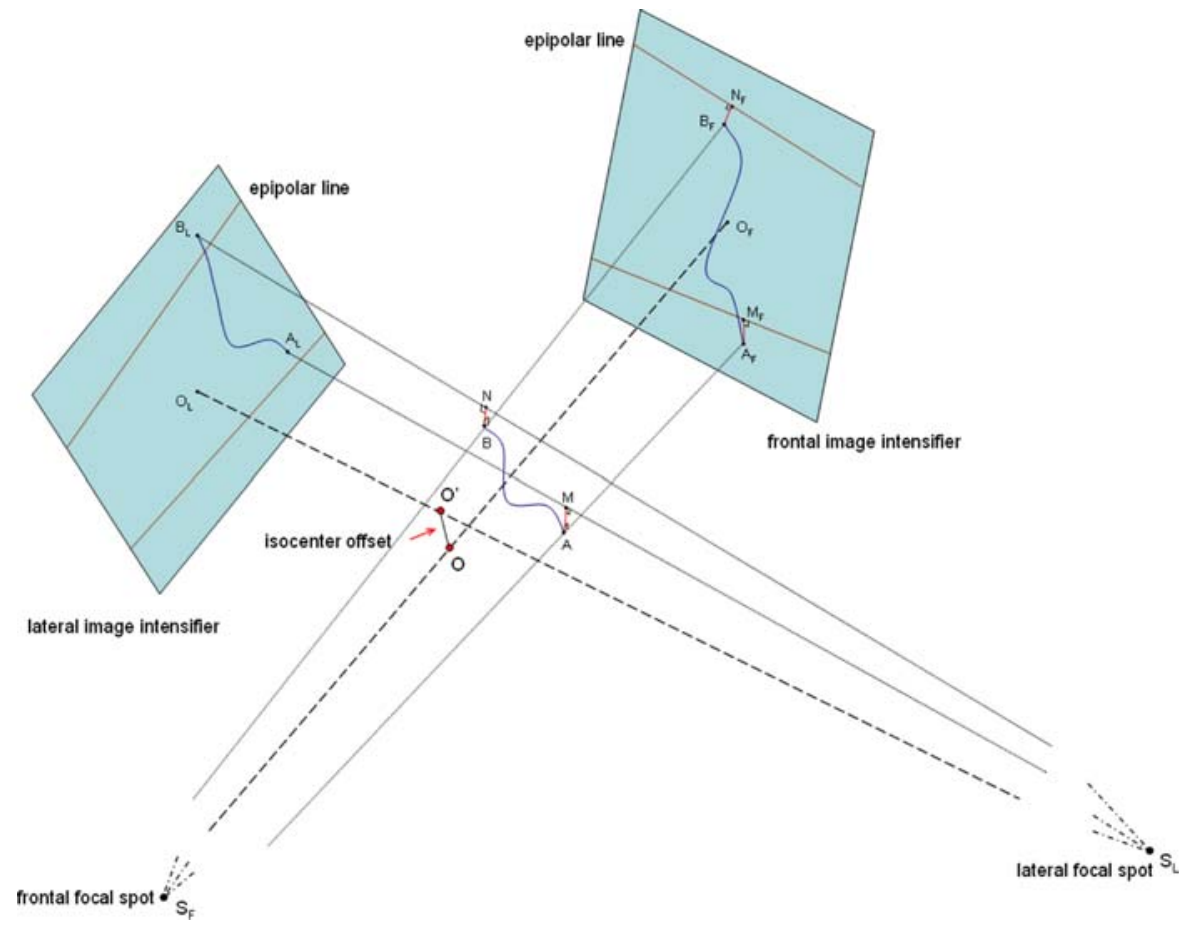

Fig. 1 3D biplane model with an isocenter offset

Fig. 2 Correspondence before correcting for the isocenter offset
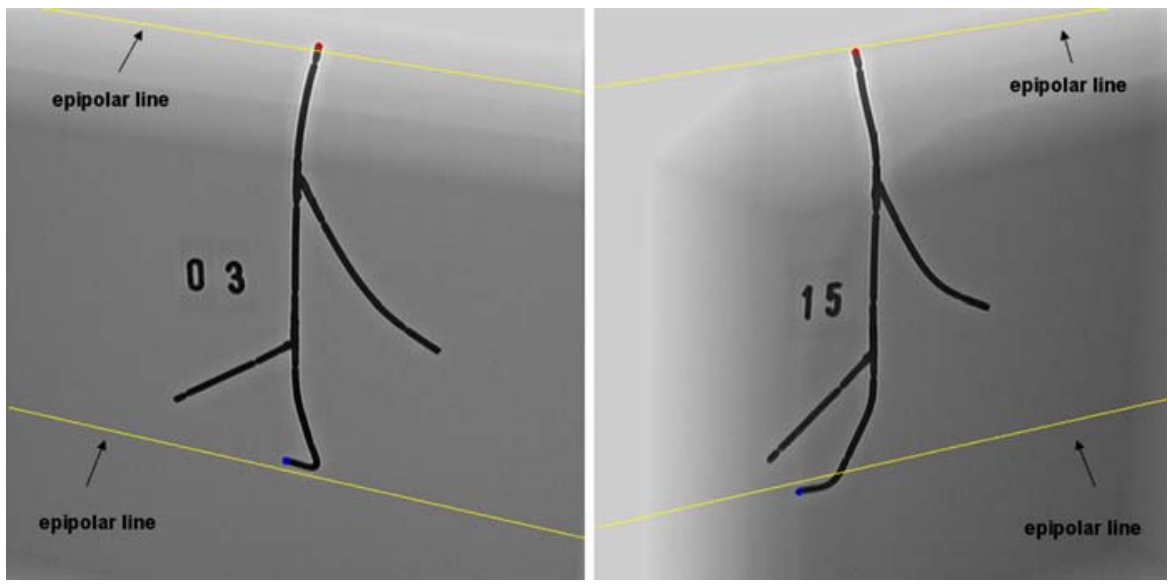

Two possible types of errors might exist in creating the correspondence between the frontal and lateral centerlines:

- The first error comes from the ill-defined epipolar lines due to the system distortion, mainly the isocenter offset. The correction of the isocenter offset in our 3D model will allow more accurate usage of the epipolar constraint in creating the correspondence, e.g., the corrected epipolar lines of the start and end points in Fig. 4 correspond better with the projection centerline than the original epipolar lines.

- The second error comes from the noise-corrupt centerlines, especially for those with low contrast background and a small perspective viewing angle, e.g., epipolar line a in Fig. 4, which could introduce quite cumbersome problems and affect the quality of correspondence.

To address the problems of using the epipolar constraint in difficult situations, a distance transform 
Fig. 3 Correspondence after correcting for the isocenter offset
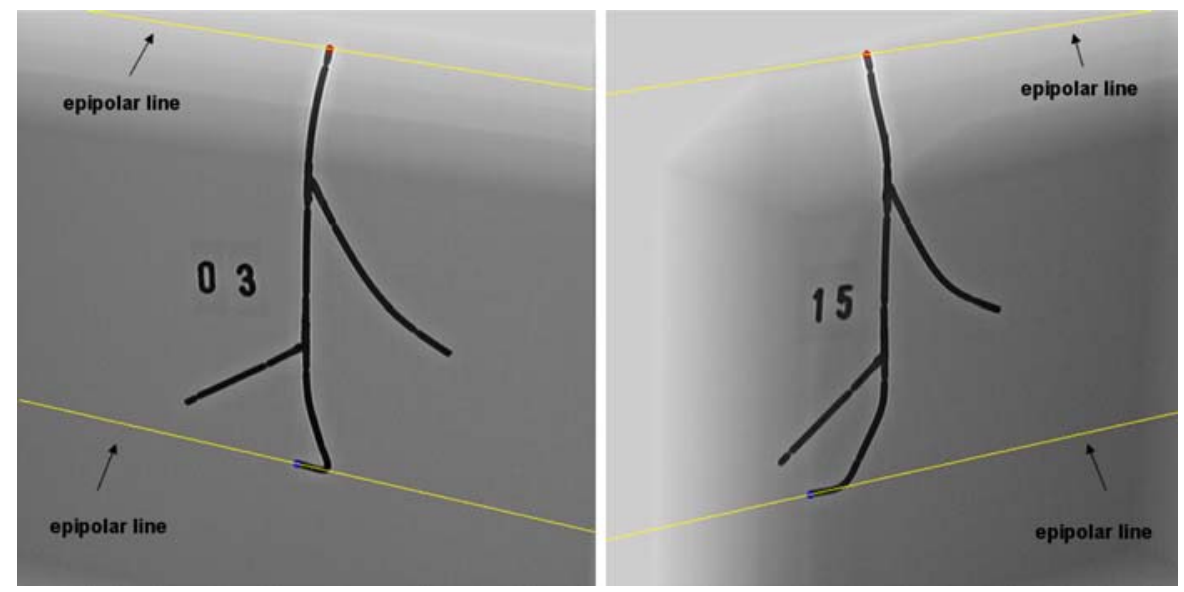

Fig. 4 Possible difficulties in corresponding two projection centerlines by using epipolar constraint

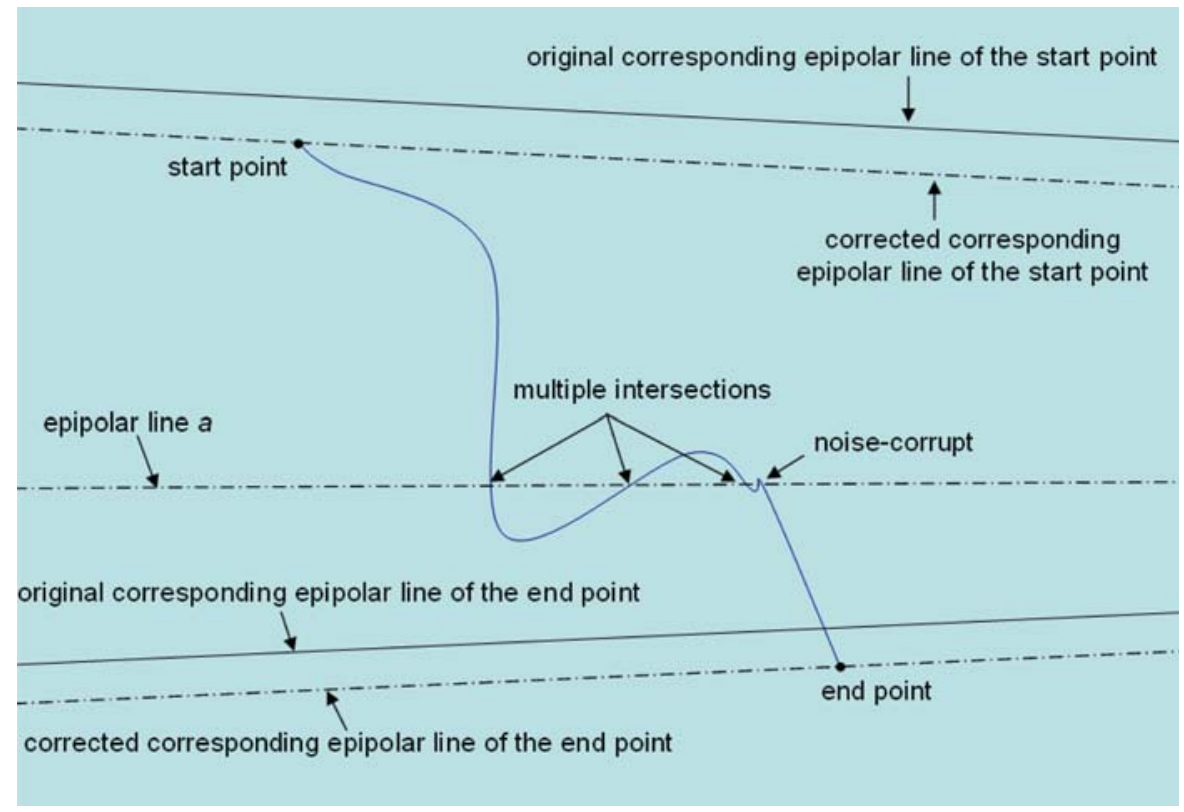

matrix is constructed based on the distance from each projection centerline point to its corresponding epipolar line. A wave propagation algorithm [9] is then applied to search for a smooth corresponding path by which the propagation from the start position to the end position has the lowest cost. Based on the correspondence path, point reconstruction will be performed on each pair of corresponding points. We adopted the point reconstruction algorithm used by Dumay and Wahle $[2,5]$. Each pair of corresponding points will associate with two projection rays. The weighted middle point of the shortest distance vector perpendicular to both projection rays is used as the approximation to the reconstruction point.

\section{Applications}

Obstruction length assessment

In coronary interventions, accurate assessment of obstruction length is of utmost importance for the selection of the appropriate stent size. The conventional approach to calculate obstruction length is to perform 2D QCA on the end-diastolic image frame $[1,10]$. After detecting the start and end positions of the obstruction, the pixel length is calculated and multiplied with the calibration factor to generate the obstruction length. Since the calibration factor only holds true for one particular plane perpendicular to 
the projection axis, e.g., the catheter plane or isocenter plane, and this procedure assumes that the obstructed vessel segment lies in that particular plane, significant error due to the out-of-plane magnification [11] could exist when the assumption is not satisfied during the image acquisition. Besides, due to the $2 \mathrm{D}$ representations of the $3 \mathrm{D}$ vascular structures, 2D QCA has inherent limitations in assessing curved segment length from vessel foreshortening. The amount of foreshortening in 2D QCA relies on the shape of vessel and the experience of the operators in choosing the so-called optimal viewing angle during the image acquisition. A significant vessel foreshortening by performing 2D QCA on the operatorselected view in standard clinical acquisition has been reported in early literatures [12-14].

Figure 5 shows an example of comparing 3D QCA and 2D QCA in assessing obstruction length. The centerline and cross sections of segment of interest were reconstructed from biplane data (frontal image under RAO $28.7^{\circ}$ and Cranial $0.3^{\circ}$, lateral image under LAO $49.2^{\circ}$ and Cranial $0.2^{\circ}$ ) and the obstructed segment was automatically detected. The obstruction start and end positions in frontal image, lateral image, and $3 \mathrm{D}$ reconstructed vessel segment were synchronized before assessment. 2D QCA was performed on both frontal and lateral images by using isocenter calibration method. A significant error, caused by vessel foreshortening and out-of-plane magnification, was noticed from 2D QCA assessments: The obstruction length was measured as $11.20 \mathrm{~mm}$ in the frontal image and $9.80 \mathrm{~mm}$ in the lateral image, respectively, while the 3D obstruction length was $14.64 \mathrm{~mm}$. The error of 2D QCA assessment in the frontal image comes predominantly from the out-of-plane magnification, since the obstructed vessel segment does not lie in the frontal isocenter plane, i.e., the plane perpendicular to the frontal projection axis and passing through the isocenter (the white intersection point of two yellow lines in Fig. 5). Since the obstructed segment is also not close to the catheter plane, the outof-plane magnification would still cause significant error, if the catheter calibration method instead of isocenter calibration method was used. The error of $2 \mathrm{D}$ QCA assessment in the lateral image is caused by the combination of out-of-magnification and vessel foreshortening, which is more significant in this case.

Optimal bifurcation viewing angle assessment

Due to the increasing complexity of coronary interventions, in particular for the intervention of bifurcation lesions, the identification of the optimal viewing angle is of increasing importance to the

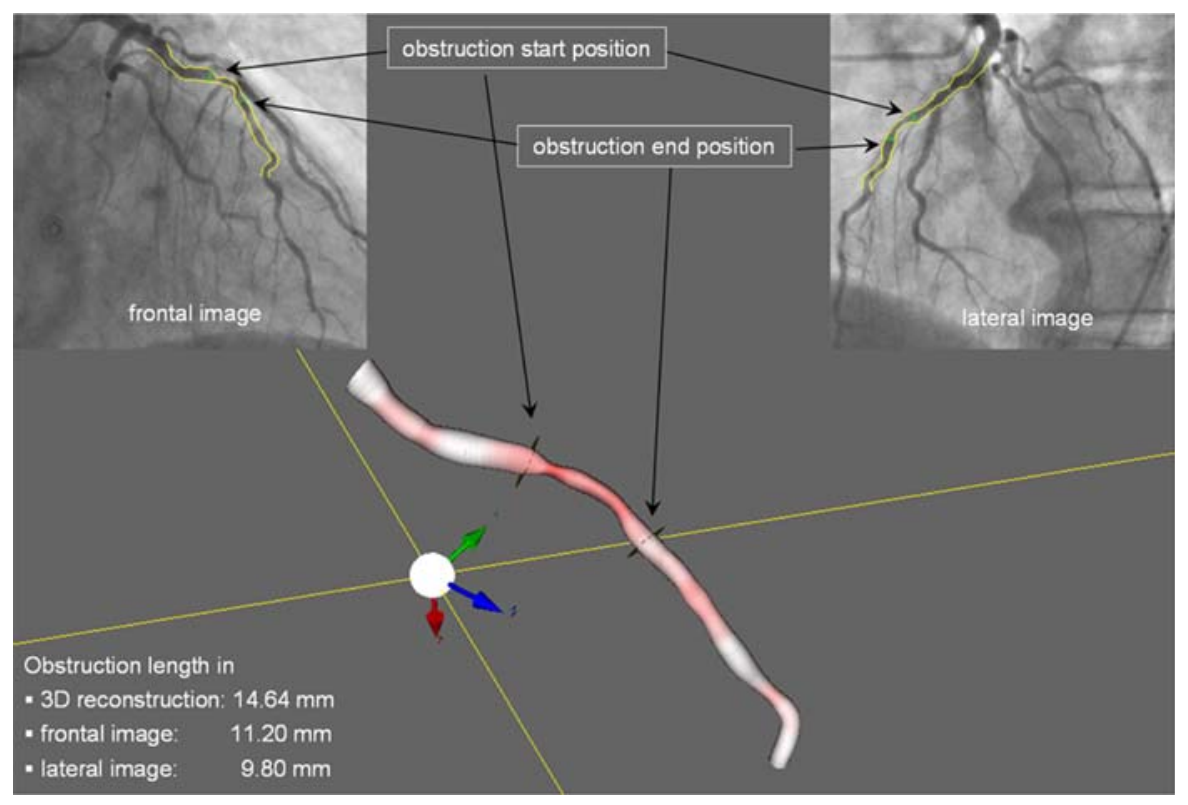

Fig. 5 Comparison of 3D QCA and 2D QCA in assessing obstruction length. Frontal image (top left) and lateral image (top right) are biplane data. Courtesy: Department of Cardiology, Leiden University Medical Center (LUMC), The Netherlands 
interventionalists for optimally deploying the balloon and stent. To stent certain types of bifurcation lesions, e.g., the one classified as $0,1,0$ according to the Medina classification [15], a sub-optimal viewing angle might not entitle the interventionalists to clearly visualize the ostium of a side branch, possibly resulting in jailing of the side branch [16]. In case of stenting the ostium of a side branch, a good viewing angle could help the interventionalists to prevent stent protrusion into the main branch or incomplete lesion coverage at the ostium of the side branch [17].

In routine clinical practice, the optimal viewing angle is subjectively selected by adjusting the rotation angle (LAO/RAO) and angulation angle (Cranial/ Caudal) of the X-ray gantry. This "trial-and-error" approach could significantly increase the amount of contrast medium administration and the radiation exposure to the patient and staff. In addition, due to the various experiences and preferences of the interventionalists, there is no guarantee that the chosen angle optimally visualizes the segment of interest. Therefore, a number of automatic methods have been developed to identify the optimal viewing angle after the 3D reconstruction. Chen et al. [18] defined the optimal viewing angle as the projection view having minimum foreshortening and overlap of a specific region in angiographic images. However, in case of a bifurcation with strongly curved main branch, the viewing angle minimizing the foreshortening of the main branch is not always the same view optimizing the visualization of the ostium of a side branch, e.g., some bifurcations in the left anterior descending arteries [16]. Besides, the choice of a specific region for calculating the foreshortening and overlap is also subjective. Christiaens et al. [19] followed the method of determining optimal viewing angle for a straight vessel by Dumay et al. [20] and defined the optimal bifurcation viewing angle as the angle perpendicular to the main direction of the bifurcation branches. Again, in a heavily curved main branch, the optimal viewing angle calculated by this approach might not work for best visualizing the ostium of the side branch in the bifurcation, where the majority of restenosis occurred following $\mathrm{T}$-stenting.

We have decided to take another approach and define a bifurcation main plane by linear regression of two centerlines within the bifurcation core, which starts from the proximal delimiter where the two centerlines start to split and ends at two distal

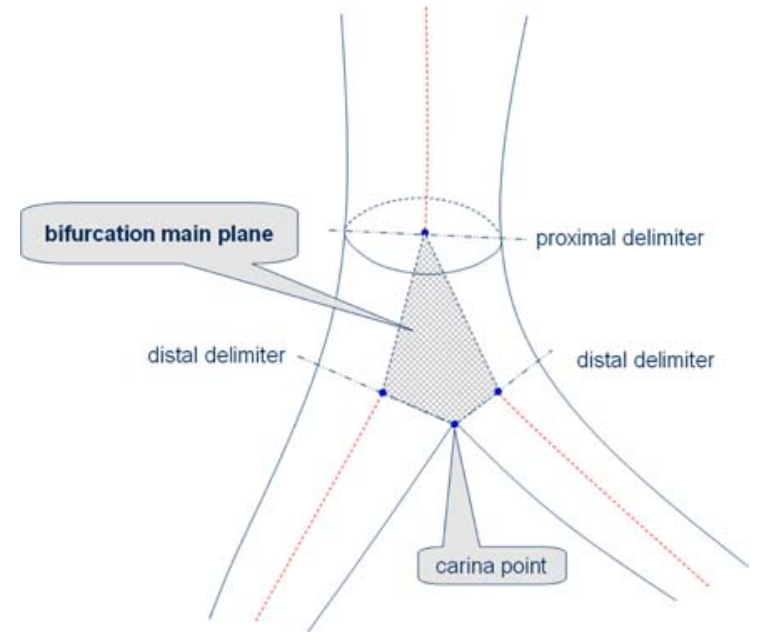

Fig. 6 The definition of bifurcation main plane

delimiters where the bifurcation core ends and separates into two distal branches, and by minimizing the distance from carina point to the plane. Figure 6 shows the definition of bifurcation main plane. The optimal viewing angle is determined by the direction perpendicular to the bifurcation main plane. By this viewing angle, the visualization of the ostium of the side branch is improved when a heavily curved main branch is present.

Figure 7 shows a clinical example of a biplane acquisition. The frontal image was acquired under RAO $35.8^{\circ}$ and Caudal $0.2^{\circ}$, while the lateral image was acquired under LAO $53.4^{\circ}$ and Caudal $0.2^{\circ}$. The start and end positions of the bifurcation were indicated for the reconstruction. Figure 8 shows the visualization of the reconstructed bifurcation under the optimal viewing angle, being LAO $52.0^{\circ}$ and Caudal $20.1^{\circ}$. Clearly, the bifurcation core and the side branch are well visualized and have minimum overlap under the optimal view. It is expected that this viewing angle will enable the interventionalists to accurately see whether the stent has completely covered the ostium of a side branch and whether there is stent protrusion into the main branch.

\section{Validations}

Data acquisition protocols

Three wire phantoms with a number of markers were used in the validation study. In the Leiden University 
Fig. 7 A biplane data: frontal image (left); lateral image (right). Courtesy: Department of Cardiology, Leiden University Medical Center (LUMC), The Netherlands
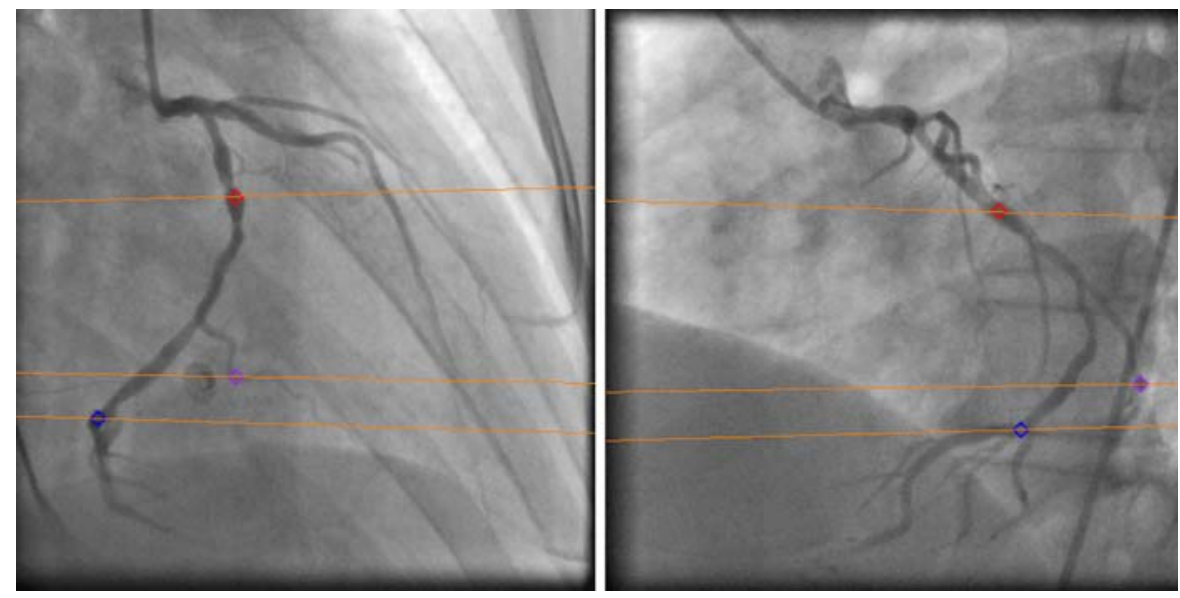

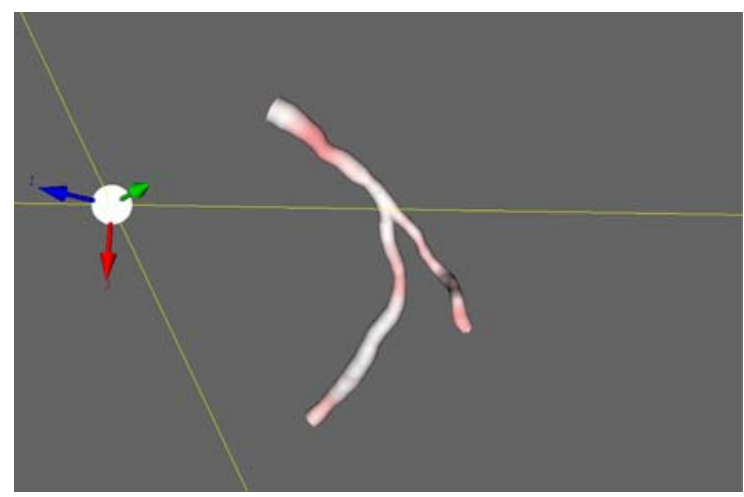

Fig. 8 The reconstructed bifurcation under the optimal viewing angle: LAO $52.0^{\circ}$ and Caudal $20.1^{\circ}$

Medical Center, data were acquired using a Toshiba biplane X-ray system with a flat screen image intensifier. The distance from the focal spot to the image intensifier was set at $1100 \mathrm{~mm}$. The first phantom was acquired with image resolution of $512 \times 512$ and intensifier size of $15 \mathrm{~cm}$, while the other two phantoms were acquired with image resolution of $1024 \times 1024$ and intensifier size of $20 \mathrm{~cm}$. All phantoms were acquired under multiple projection angles and images were stored in DICOM files. Figure 9 shows two of the wire phantoms used in the validation study. The thin cutting positions on the wires were used as markers.

\section{Segment length assessment}

Twelve segments with length ranging from 16.5 to $39.0 \mathrm{~mm}$ were defined by the markers on the wire phantoms. The average length for these 12 segments is $24.15 \mathrm{~mm}$. Each segment was reconstructed four to five times using different pairs of acquisitions (with a difference of $30^{\circ}$ to $120^{\circ}$ in acquisition angles between the frontal and lateral projections) and its length was measured from each reconstruction, leading to 52 QCA measurements. The accuracy of these measurements was assessed by comparing these with the known true length of the wire segments.

\section{Optimal bifurcation viewing angle}

In order to determine the ground truth of optimal viewing angle for each bifurcation, two orthogonal iron sticks were attached to each bifurcation, one stick on the main distal branch and the other one on the side branch, with the first half parts of two iron sticks joining together as the optimal viewing vector. Figure 10 shows two projections of one wire phantom with the attached orthogonal iron sticks. The optimal viewing vector for each bifurcation was carefully adjusted to the best direction to view its related bifurcation. After that, the phantom was put back to the same position on the table of the X-ray system as the previous acquisitions. For each bifurcation, the table was moved so that the bifurcation core was visualized in the middle of the projection image. Next, the rotation and angulation angles were adjusted until the optimal viewing vector was visualized as one point. The reading of the acquisition angles was used as the ground truth for that particular bifurcation. An example of the projections from the phantom under the optimal viewing angle for the lowest bifurcation (arrow in Fig. 10a) and the middle bifurcation (arrow in Fig. 10b) is given in Fig. 10. 
Fig. 9 Wire phantoms used in the validation study

Fig. 10 Determining the ground truth of optimal viewing angle by using the orthogonal iron sticks: left image (a) under RAO $4.0^{\circ}$ and Cranial $40.0^{\circ}$; right image (b) under LAO $44.0^{\circ}$ and Cranial $3.0^{\circ}$. The arrow indicates which bifurcation is optimally visualized
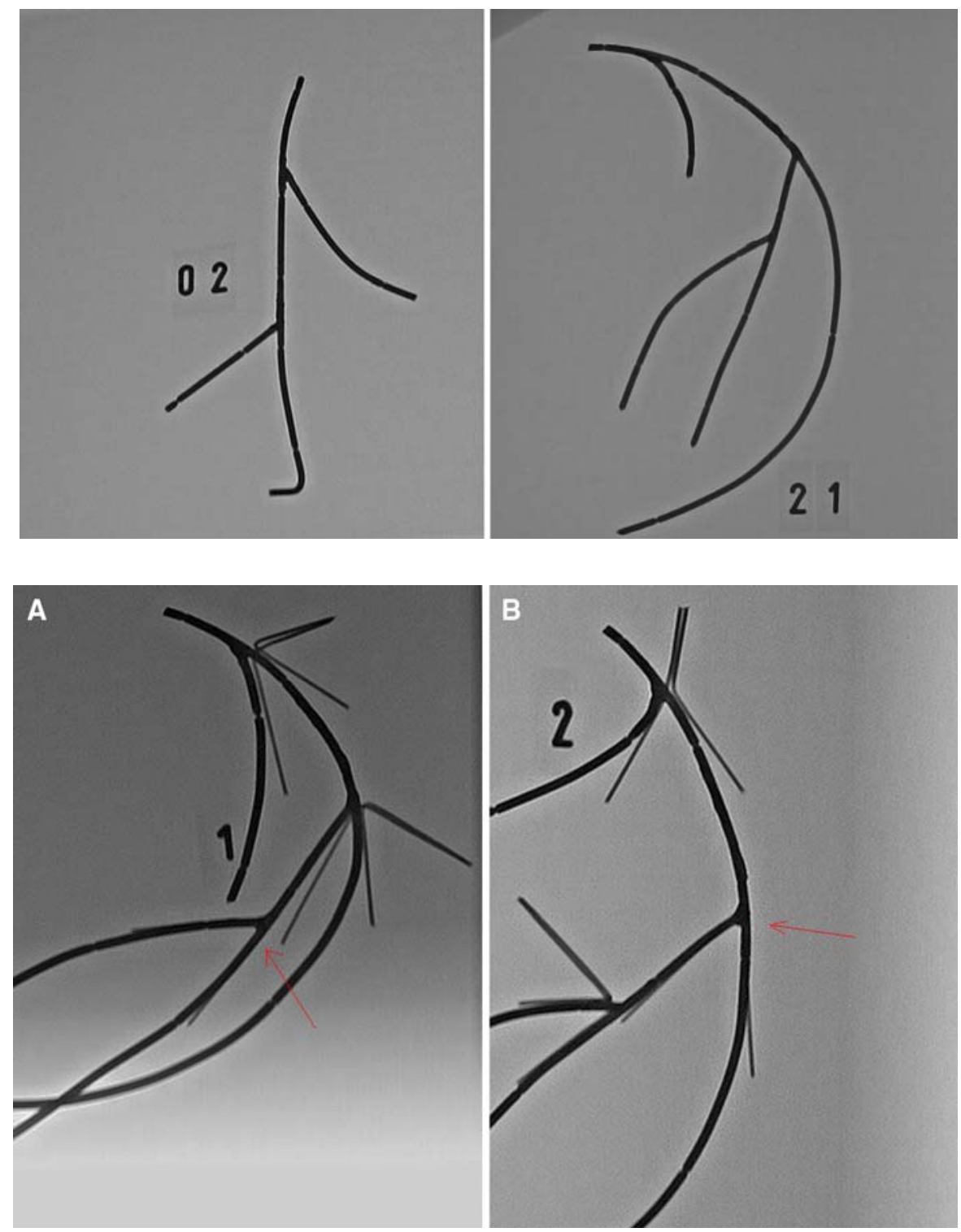

In total six bifurcations from three wire phantoms were used in the validation. Each bifurcation was reconstructed eight times using different pairs of acquisitions (with a difference of $30^{\circ}$ to $120^{\circ}$ in acquisition angles between the frontal and lateral projections) and its optimal viewing angle was assessed from the reconstruction, leading to 48 assessments.

\section{Statistics}

The correlation between 3D QCA segment length and the true wire segment length was calculated using
Pearson's correlation coefficient. The Bland-Altman plot was used to evaluate the difference between the 3D QCA assessment and the true length, while student $t$ test was performed to investigate the statistical significance of the difference.

The difference of optimal viewing angles between the 3D QCA assessment and the ground truth was evaluated by a scatter plot in terms of rotation angle and angulation angle. The mean difference of the optimal viewing angle was computed and considered to be an index to the accuracy of the QCA assessment, while the standard deviation of the difference was considered as an index of precision. Student $t$ test 
was performed to investigate the statistical significance of the difference.

All statistical analyses were carried out by using statistical software (SPSS, version 16.0; SPSS Inc.; Chicago, IL, USA).

\section{Results}

The correlation of 3D QCA segment length and the true wire segment length is presented in Fig. 11. Clearly, the segment length assessed by 3D QCA correlated very well with the true wire segment length $\left(r^{2}=0.999\right)$. Bland-Altman plot for the correlation is given in Fig. 12. No trend for the

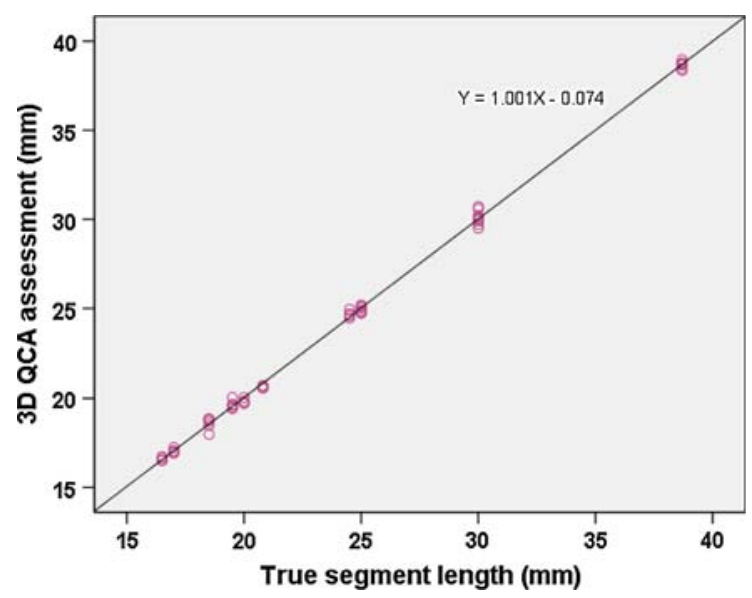

Fig. 11 Correlation of 3D QCA segment length and the true wire segment length

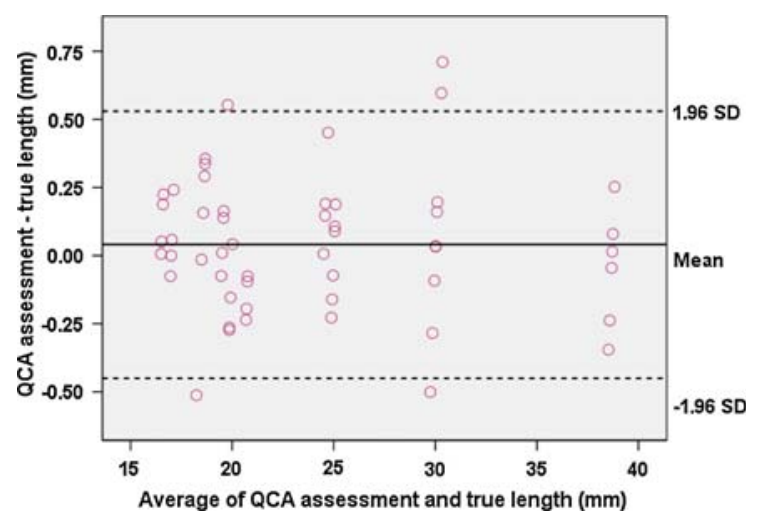

Fig. 12 Bland-Altman plot of 3D QCA segment length and the true wire segment length difference as a function of the true length was found. The mean and standard deviation of the difference between QCA assessment and the true length were 0.04 and $0.25 \mathrm{~mm}$, respectively. The difference was significant $(P<0.01)$, in other words, 3D QCA slightly overestimated the segment length by $0.04 \mathrm{~mm}$ for a segment with average length of $24.15 \mathrm{~mm}$.

An optimal viewing angle consists of two parts: rotation angle and angulation angle. The scatter plot for the difference of optimal viewing angle assessed by $3 \mathrm{D}$ QCA and the ground truth in terms of these two parts is given by Fig. 13. The shape of the scatter points represents the bifurcation case. No specific pattern was observed within any bifurcation case, indicating that the assessment was not sensitive to the acquisition angles for the reconstruction. The descriptive statistics is given by Table 1 . The mean and standard deviation of the difference of rotation angles between QCA assessment and the ground truth was $-1.5^{\circ}$ and $3.6^{\circ}$, respectively. The difference was significant $(P<0.01)$. The mean and standard deviation of the difference of angulation angles between 3D QCA assessment and the ground truth was $-0.2^{\circ}$ and $2.4^{\circ}$, respectively. The difference was not significant $(P=0.54)$. In other words, 3D QCA slightly underestimated the optimal rotation angle by $1.5^{\circ}$.

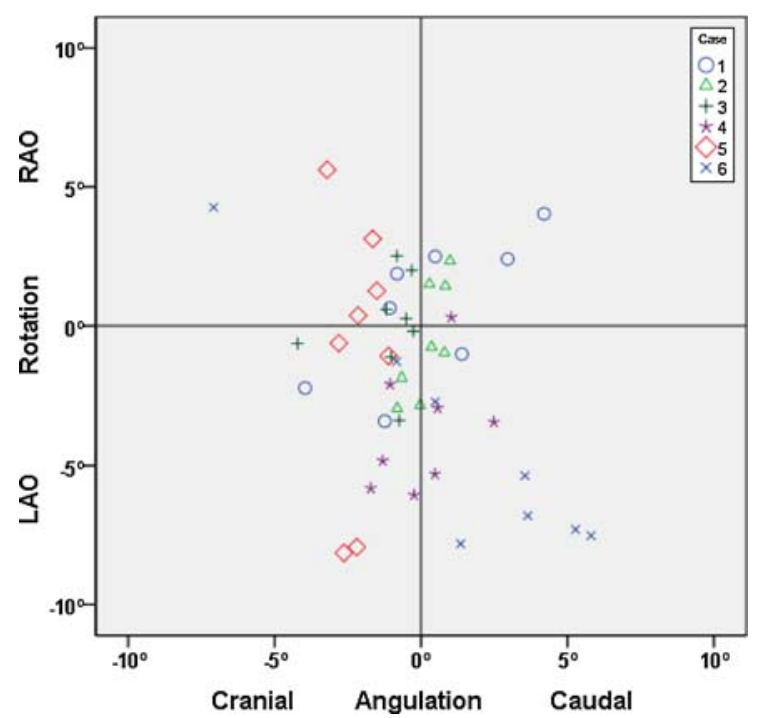

Fig. 13 Scatter plot for the difference of optimal view angle between 3D QCA assessment and the ground truth 
Table 1 The difference of optimal viewing angle between QCA assessment and the ground truth

\begin{tabular}{llllr}
\hline & Number of assessment & Minimum & Maximum & Mean \\
\hline Rotation (RAO) & 48 & $-8.1^{\circ}$ & $5.6^{\circ}$ & $-1.5^{\circ}$ \\
Angulation (CAUD) & 48 & $-7.1^{\circ}$ & $5.8^{\circ}$ & $3.6^{\circ}$ \\
\hline
\end{tabular}

\section{Discussions}

Over the past years, the development of coronary visualization and quantitative analysis systems has been motivated by the increasing need to better understand the true dimensions of vascular structures and by the on-line need for coronary intervention in catheterization laboratories. 3D QCA has received a lot of interest on the potential benefits for increasing the assessment capabilities for both diagnostic and interventional cardiology. It was thought that the 3D QCA could resolve a number of additional limitations of standard 2D analysis, such as elimination of foreshortening and out-of-plane magnification error [11] in obstruction length assessment. In addition, the automatic identification of the optimal viewing angle would benefit interventionalists and patients from less radiation exposure by reducing the trials in achieving the best "working view".

In spite of the fact that two simultaneously acquired images are available from biplane X-ray imaging systems, the development of a reliable and robust 3D QCA system is still not a trivial task. All current 3D QCA systems work best under conditions of the two X-ray systems rotating around the isocenter. However, the change of gantry geometry during the image acquisition might significantly shift the isocenter. In addition, the requirement of rotating two X-ray gantries around the same isocenter is a significant constraint to the operator in clinical routine. In other words, 3D QCA should also work accurately under non-isocentric conditions. In order to achieve this, the isocenter offset, i.e., the difference between the isocenters of the frontal and lateral gantries, should be approximated and corrected before the reconstruction. Ideally, a couple of reliable features should be identified on both projections as reference points for correcting for the isocenter offset, on the other hand, the practical usage has been hampered by the effort in identifying many reliable features, which turned out to be too time consuming or even impossible to identifying such reliable features on both projections. We have developed an approach by using only one to three pairs of reference points for correcting for the isocenter offset. Phantom validation by using only one or two markers as reference points for correcting for the isocenter offset showed a high accuracy on the assessments of segment length and optimal viewing angle. In addition to the refinement of imaging geometry, we have also addressed the difficult problems in centerline reconstruction when small perspective projection angles and noise-corrupt centerlines are present, which are expected to occur more in clinical acquisitions. Although different acquisitions were used for the reconstruction (The difference of acquisition angles for frontal and lateral projections varies from $30^{\circ}$ to $120^{\circ}$ ), the variations of the assessments for both segment length and optimal viewing angle were relatively small.

The delineation of vessel segment in 3D QCA could potentially increase the accuracy in stent selection. In current approaches, the selection of stent sizes mainly depends on the obstruction length assessed by visual estimation (eyeballing) or by performing 2D QCA. Conventionally, calibration procedure, e.g., catheter calibration, should be performed at the first step of the assessment, which might as well introduce calibration error. In addition, the foreshortening of the vessel of interest could cause significant underestimation of segment length [13, 21, 22], which could not be assessed or recognized directly from the $2 \mathrm{D}$ projection images. It happens quite some times when an inexperienced interventionalist chooses a stent which is too short and then ends up by putting in another stent. In some catheterization laboratories it becomes common for the interventionalists to consider the obstruction length a bit longer than the assessed result. As a result of that, the selection of stent might turn out to be longer than necessary, which could change unnecessarily the behavior of the arteries and associate a possible high rate of restenosis. On the other hand, the usage of automatic calibration in 3D QCA 
and the high accuracy of 3D QCA in segment length assessment could change the operator in decision making [12] and offer more benefits to the patients. Although we only validate the segment length, it is reasonable to expect that the accuracy of assessment will hold for the obstruction length, based on the highly accurate correspondence between two projections achieved by our system. Once a criterion has been applied to the definition of obstruction, e.g., more than $50 \%$ percent stenosis, the obstruction length will be accurately assessed.

The ability to identifying the optimal viewing angle is another important feature of 3D QCA systems, especially for supporting the on-line coronary intervention. Nevertheless, the optimal viewing angle has been interpreted differently: optimal viewing angle with minimal foreshortening and overlap [3], optimal viewing angle for best visualizing the severity of lesion, or optimal viewing angle for best visualizing stent position in the bifurcation. These interpretations might result in different outcomes for certain kinds of bifurcations, e.g., the left main bifurcation with strongly curved left anterior descending artery. For the best interest of bifurcation related interventions, we have decided to taken the last interpretation and we believe that a good visualization of the bifurcation main plane could significantly benefit the interventionalists in deploying the bifurcation stent and increase the angiographic success. An example case can be observed in T-Stenting [23]: inappropriate view of the stent position might lead to incomplete lesion covering at the ostium of a side branch or stent protrusion into the main vessel during the deployment of the stent.

Despite high accuracy and robustness have been achieved on our 3D QCA system, the practical usage of the system has been hampered by the fact that biplane X-ray angiogram is hardly used as clinical routine in interventional cardiology. However, combing with the ECG-gated technique, our approach can be extended with a solution for monoplane X-ray system. The introduction of isocenter offset correction could also be expected to eliminate the shift of heart caused by the patient respiration when changing the gantry from the first projection angle to the second projection angle. Future work is directed at performing extensive clinical validations for monoplane acquisition systems.

\section{Conclusions}

A novel 3D QCA system based on X-ray angiograms has been achieved by introducing a highly reproducible vessel centerline reconstruction. The validation study by using wire phantoms showed a high degree of accuracy and precision on the assessments of segment length and optimal viewing angle.

Open Access This article is distributed under the terms of the Creative Commons Attribution Noncommercial License which permits any noncommercial use, distribution, and reproduction in any medium, provided the original author(s) and source are credited.

\section{References}

1. Reiber JHC, Koning G, Tuinenburg JC, Lansky A, Goedhart B (2004) Quantitative coronary arteriography. In: Oudkerk M (ed) Coronary radiology. Springer, Berlin, pp 41-58

2. Wahle A, Oswald H, Fleck E (1996) 3D heart-vessel reconstruction from biplane angiograms. IEEE Comput Graph Appl 16(1):65-73

3. Chen SJ, Carroll JD, Messenger JC (2002) Quantitative analysis of reconstructed $3-\mathrm{D}$ coronary arterial tree and intracoronary devices. IEEE Trans Med Imaging 21(7): 724-740

4. Metz CE, Fencil LE (1989) Determination of threedimensional structure in biplane radiography without prior knowledge of the relationship between the two views: theory. Med Phys 16:45-51

5. Dumay ACM (1992) Image reconstruction from biplane angiographic projections. Dissertation, Delft University of Technology

6. Hoffmann KR, Anindya S, Li L et al (2000) A system for determination of $3 \mathrm{D}$ vessel tree centerlines from biplane images. Int J Card Imaging 16(5):315-330

7. Wahle A, Wellnhofer E, Mugaragu I, Sauer HU et al (1995) Assessment of diffuse coronary artery disease by quantitative analysis of coronary morphology based upon 3 -D reconstruction from biplane angiograms. IEEE Trans Med Imaging 14(2):230-241

8. Van Der Zwet PMJ, Reiber JHC (1994) A new approach for the quantification of complex lesion morphology: the gradient field transform; basic principle and validation results. J Am Coll Cardiol 24(1):216-224

9. Janssen JP, Koning G, de Koning PJH, Tuinenburg JC, Reiber JHC (2002) A novel approach for the detection of pathlines in X-ray angiograms: the wave propagation algorithm. Int J Cardiovasc Imaging 18:317-324

10. Goktekin O, Kaplan S, Dimopoulos K et al (2007) A new quantitative analysis system for the evaluation of coronary bifurcation lesions: comparison with current conventional methods. Catheter Cardiovasc Interv 69:172-180

11. Koning G, Hekking E, Kemppainen JS, Richardson GA, Rothman MT, Reiber JHC (2001) Suitability of the cordis 
stabilizer $^{\mathrm{TM}}$ marker guide wire for quantitative coronary angiography calibration: an in vitro and in vivo study. Catheter Cardiovasc Interv 52(3):334-341

12. Gollapudi RR, Valencia R, Lee SS, Wong GB, Teirstein PS, Price MJ (2007) Utility of three-dimensional reconstruction of coronary angiography to guide percutaneous coronary intervention. Catheter Cardiovasc Interv 69: 479-482

13. Agostoni P, Biondi-Zoccai G, Van Langenhove $G$ et al (2008) Comparison of assessment of native coronary arteries by standard versus three-dimensional coronary angiography. Am J Cardiol 102(3):272-279

14. Green NE, Chen S-YJ, Hansgen AR, Messenger JC, Groves BM, Carroll JD (2005) Angiographic views used for percutaneous coronary interventions: a three dimensional analysis of physician-determined vs. computergenerated views. Catheter Cardiovasc Interv 64:451-459

15. Medina A, de Lezo JS, Pan M (2006) A new classification of coronary bifurcation lesions. Rev Esp Cardiol 59(2):183-184

16. Sadamatsu K, Sagara S, Yamawaki T, Tashiro H (2009) Three-dimensional coronary imaging for the ostium of the left anterior descending artery. Int $\mathbf{J}$ Cardiovasc Imaging 25(3):223-228

17. Lemos PA, Saia F, Ligthart JM et al (2003) Coronary restenosis after sirolimus-eluting stent implantation: morphological description and mechanistic analysis from a consecutive series of cases. Circulation 108:257-260

18. Chen SJ, Carroll JD (2000) 3-D reconstruction of coronary arterial tree to optimize angiographic visualization. IEEE Trans Med Imaging 19(4):318-336

19. Christiaens J, Van R, de Walle P, Gheeraert YT, Lemahieu I (2001) Determination of optimal angiographic viewing angles for QCA. Int Congr Ser 1230:909-915

20. Dumay ACM, Reiber JHC, Gerbrands JJ (1994) Determination of optimal angiographic viewing angles: basic principles and evaluation study. IEEE Trans Med Imaging 13(1):13-24

21. Bruining N, Tanimoto S, Otsuka M et al (2008) Quantitative multi-modality imaging analysis of a bioabsorbable poly-L-lactic acid stent design in the acute phase: a comparison between 2- and 3D-QCA, QCU and QMSCT-CA. EuroIntervention 4:285-291

22. Rittger H, Schertel B, Schmidt M, Justiz J, Brachmann J, Sinha A (2009) Three-dimensional reconstruction allows accurate quantification and length measurements of coronary artery stenoses. EuroIntervention 5(1):127-132

23. Vigna C, Biondi-Zoccai G, Amico CM et al (2007) Provisional T-drug-eluting stenting technique for the treatment of bifurcation lesions: clinical, myocardial scintigraphy and (late) coronary angiographic results. J Invasive Cardiol 19(3):92-97 